\title{
Prevalencia de sucesos violentos y de trastorno por estrés postraumático en la población mexicana
}

\author{
Ma. Elena Medina-Mora Icaza, Dra en Psic Soc, ${ }^{(1)}$ Guilherme Borges-Guimaraes, Dr en SP,(2) \\ Carmen Lara, Dra en $C_{,}{ }^{(3)}$ Luciana Ramos-Lira, Dra en Psic Soc, ${ }^{(4)}$ Joaquín Zambrano,Actuario, ${ }^{(4)}$ \\ Clara Fleiz-Bautista, Lic en Psic.(4)
}

\begin{abstract}
Medina-Mora ME, Borges-Guimaraes G, Lara C, Ramos-Lira L, Zambrano J, Fleiz-Bautista C. Prevalencia de sucesos violentos y de trastorno por estrés postraumático en la población mexicana. Salud Publica Mex 2005;47:8-22. El texto completo en inglés de este artículo está disponible en: http://www.insp.mx/salud/47/eng
\end{abstract}

\section{Resumen}

Objetivo. Reportar el índice de exposición a diferentes sucesos violentos, los correlatos demo gráficos, la prevalencia de trastorno por estrés postraumático y el impacto sobre la calidad de vida. Material y métodos La Encuesta $\mathrm{N}$ acional de Epidemio logía Psiquiátrica es representativa de la población mexicana urbana de 18 a 65 años de edad. Se realizó entre 2001 y 2002, con el instrumento diagnóstico de la versión computarizada de la Entrevista Internacional Compuesta de Diagnóstico (CIDI-15, por sus siglas en inglés). Los análisis toman en cuenta el diseño complejo de la muestra aleatoria, multietápica y estratificada. Se utilizaron el Método Kaplan-Meir y regresiones logísticas. Resulta dos. El $68 \%$ de la población ha estado expuesta al menos a un suceso estresante en su vida. La exposición varía por sexo (violación, acoso y abuso sexual son más frecuentes en mujeres; los accidentes y robos, entre los hombres) y por edad (niños, adolescentes, mujeres adultas jóvenes y
Medina-Mora ME, Borges-Guimaraes G, Lara C, Ramos-Lira L, Zambrano J, Fleiz-Bautista C. Prevalence of violent events and post-traumatic stress disorder in the Mexican population. Salud Publica Mex 2005;47:8-22.

The English version of this paper

is available at: http://www.insp. mx/salud/47/eng

\section{A bstract}

Objective.To report the rate of exposure to different violent events, their demographic correlates, the prevalence of Post-Traumatic Stress D isorder (PTSD), and the impact on quality of life. Material and Methods. The $\mathrm{N}$ ational Survey of Psychiatric Epidemiology is representative of the Mexican urban population aged 18 to 65 . The survey was undertaken in 2001 and 2002 using the Composite International Diagnostic Inter view (CIDI-15) computerized version. The statistical analyses take into account the multistage, stratified, and weighted sample design. Kaplan-Meier and logistic regressions were performed. Results. Sixty-eight percent of the population has been exposed to at least one stressful life event. Exposure varies by sex (rape, harassment, and sexual abuse are more frequent in women; accidents and being a victim of burglary among men) and by age (more frequent in children, ado lescents, young adult women, and the elderly). By sex, $2.3 \%$ of women and $0.49 \%$ of

Este trabajo recibió apoyo financiero del Instituto Nacional de Psiquiatría Ramón de la Fuente, proyecto N 0.4280, del Consejo Nacional de Ciencia y Tecnología, proyecto N o. G30544-H, de la O rganización Panamericana de la Salud, y de Pfizer México.

La investigación base de la que provienen los datos forma parte de la Iniciativa 2002 de la 0 rganización Mundial de la Salud, en Salud Mental, con apoyo del $\mathrm{N}$ ational Institute on Mental Health (N IMH) y de la 0 rganización Panamericana de la Salud. En México, los datos fueron recabados con el apoyo financiero del Instituto N acional de Psiquiatría Ramón de la Fuente, proyecto N 0.4280, y del Consejo N acional de Ciencia y Tecnología, proyecto N 0. G 30544-H.

(1) Dirección de Investigación Epidemiológica y Psicosocial, Instituto N acional de Psiquiatría Ramón de la Fuente. México, DF, México.

(2) Universidad Autónoma Metropolitana, Unidad Xochimilco, Instituto N acional de Psiquiatría Ramón de la Fuente, México, DF, México.

(3) Universidad Autónoma de Puebla, Puebla, México.

(4) Dirección de Investigación Epidemiológica y Psicosocial. Instituto N acional de Psiquiatría Ramón de la Fuente, México, D F, México.

Fecha de recibido: 1 de septiembre de 2004 • Fecha de aprobado: 10 de noviembre de 2004 Solicitud de sobretiros: Dra. Ma. Elena Medina-Mora Icaza. Calzada México-Xochimilco 101, México, DF, México. Correo electrónico: medinam@ imp.edu.mx 
personas de la tercera edad). El $2.3 \%$ de las mujeres y $0.49 \%$ de los hombres presentaron un trastorno de estrés postraumático. La violación, el acoso, el secuestro y el abuso sexual son los sucesos con mayor manifestación de trastornos por estrés postraumático. Conclusiones Los resultados refuerzan la necesidad de ampliar la cobertura de tratamiento para atender las secuelas de la violencia, considerando las importantes variaciones de género y estadios de desarrollo. El texto completo en inglés de este artículo está disponible en: http://www.insp.mx/salud/47/eng

Palabras clave: violencia; trastorno por estrés postraumático; calidad de vida; México men present PTSD. Rape, harassment, kidnapping, and sexual abuse are the events most associated with PTSD. Conclusions. The results suggest the need to increase treatment coverage to attend the consequences of violence, taking into consideration the important gender and age variations. The English version of this paper is available at: http:// ww w.insp.mx/salud/47/eng

Key words: violence; post traumatic stress disorder; quality of life; Mexico
E s de todos conocido el elevado índice de violencia a la que está expuesta la población mexicana, directa o indirectamente, y tanto en el ámbito público como en el privado. Asimismo, otros problemas como los accidentes y los desastres son también motivo de preocupación por su impacto en términos de mortalidad y morbilidad. Sin embargo, poco se sabe acerca de la proporción en la que ocurren estos sucesos en la población general del país y sobre su impacto en la salud mental de las personas expuestas.

Las consecuencias de la violencia varían en su expresión e incluyen trastornos severos como la depresión mayor, la ansiedad generalizada y el trastorno por estrés postraumático (TEPT). ${ }^{1,2}$ Otras manifestaciones incluyen problemas en el funcionamiento social, síntomas somáticos y problemas psicosociales que pueden persistir durante muchos años y afectar la calidad de vida de las personas, sin que llegue a manifestarse un síndrome de estrés postraumático. ${ }^{3}$

La Organización Mundial de la Salud (OMS) 4 subdivide a la violencia interpersonal en dos grandes categorías: la violencia familiar y de pareja, que suele ocurrir, aunque no siempre, en el hogar; y la violencia comunitaria, cometida frecuentemente fuera del hogar por individuos que pueden tener o no alguna relación con sus víctimas. Otras formas de violencia la constituyen la derivada del crimen organizado y los efectos a la exposición a los desastres naturales o los producidos por el hombre. A continuación se ofrece un breve panorama sobre algunos datos relacionados con sucesos susceptibles de generar reacciones postraumáticas.

Violencia delictiva común. Se estima que la incidencia delictiva a escala nacional ha mantenido una tendencia relativamente estable en el lapso de enero de 1997 a junio de 2002; entre 104592 a 130492 delitos del fuero común se han cometido mensualmente, con una media de 117490 y una variabilidad media de 5925 delitos. Cabe señalar que el problema delictivo se ha acentuado en las zonas urbanas; ${ }^{5}$ según la primera Encuesta Nacional sobre Inseguridad (ENSI-1) realizada en 2002, tres de cada cuatro delitos ocurrieron en éstas. ${ }^{6}$

Por su parte, la Segunda Encuesta Nacional sobre Inseguridad (ENSI-2) reporta a los robos como el delito más frecuente, destacándose que aproximadamente uno de cada dos delitos se cometieron con violencia, ya sea utilizando agresiones físicas, objetos de diverso tipo e incluso armas de fuego; por su alto contenido violento los robos o asaltos, los secuestros exprés y el abuso sexual, entre otros. ${ }^{7}$

Violencia de pareja. La Encuesta Nacional sobre Violencia contra las Mujeres, 2003, ${ }^{8}$ realizada en servicios de salud de instituciones del sector público del primer y segundo nivel de atención, reporta una prevalencia de violencia de la pareja actual de $21.5 \%$, mientras que una de cada tres mujeres ha sufrido violencia de pareja alguna vez en la vida. El tipo de violencia más frecuente fue la psicológica con $19.6 \%$, la física con $9.8 \%$, la sexual con $7 \%$ y la económica con $5.1 \%$.

Violencia sexual. Otras formas de violencia que suelen tener graves secuelas son el abuso sexual y la violación. ${ }^{9}$ La prevalencia de abuso sexual ha sido estimada en $4.3 \%$ en adolescentes varones y mujeres, estudiantes de enseñanza media y media superior de poblaciones rurales y urbanas del país, y su impacto es alto en los índices de abuso de sustancias ${ }^{10} \mathrm{y}$ en los de depresión. ${ }^{11}$

Situaciones de desastre. Tapia y colaboradores ${ }^{12}$ en un análisis del impacto de los sismos de 1985 en la po- 
blación que se ubicaba en albergues en la Ciudad de México, reportaron que 32\% de la población estudiada presentaba síntomas de estrés postraumático como angustia generalizada, agitación, temblor, dificultad para concentrarse, trastornos del sueño (insomnio y pesadillas) y alteraciones psicofisiológicas asociadas. Estos síntomas se presentaron hasta un mes después del terremoto.

Estos antecedentes nos hablan de la importancia de conocer la magnitud del problema en México, con el fin plantear, tanto estrategias de prevención de las secuelas a mediano y a largo plazo ante la exposición a sucesos violentos, como necesidades específicas de servicios y tratamiento. En este artículo se comunican por primera vez resultados de una encuesta nacional en la que se analiza la exposición a un número amplio de sucesos, se estima el establecimiento del TEPT y su impacto en la vida laboral, familiar y personal de las personas expuestas. Se utilizaron los criterios diagnósticos definidos por el Manual Diagnóstico y Estadístico de las Enfermedades Mentales (DSM-IV, por sus siglas en inglés) y los datos se obtuvieron mediante instrumentos diagnósticos estructurados y validados internacionalmente; se analizan los costos para la sociedad, medidos en términos de dificultad para llevar a cabo actividades de la vida diaria y pérdidas laborales.

El trastorno por estrés postraumático es una respuesta sintomatológica que una persona desarrolla después de haber estado expuesta a un suceso altamente estresante, debido a que ha amenazado su integridad física o su vida, o la de otras personas. La violencia sexual, los de ataques físicos, los asaltos, los secuestros, el abuso sexual infantil, el ser testigo de la muerte o de lesiones graves a otra persona por un asalto o riña, y el saber de la muerte o del asalto violento a un familiar o amigo cercano, son sucesos estresantes que el DSM-IV menciona como susceptibles de producir TEPT.

Si la persona expuesta a estos sucesos responde con miedo intenso, impotencia o terror, puede pensarse en la presencia de este diagnóstico. Asimismo, requiere la presencia de síntomas característicos: rexperimentación persistente del suceso (flashbacks o pesadillas que reproducen el hecho en cuestión, reacciones físicas y/o psicológicas ante estímulos internos que se asocian con el suceso); evitación persistente de hechos, personas, situaciones o pensamientos asociados con el suceso traumático o reducción en la capacidad de vincularse con otras personas y experimentar emociones; síntomas que manifiestan una hiperexcitación fisiológica (como problemas para dormir, irritabilidad e hipervigilancia). Esta respuesta se considera patológica si dura al menos un mes y produce malestar clínicamente significativo o empeoramiento en el funcionamento cotidiano. ${ }^{13}$

El pronóstico varía en gran medida según el tipo de estresor que generó la respuesta traumática; lo mismo ocurre con su evolución. ${ }^{14}$ En muchos casos el trastorno presenta, además, una alta comorbilidad con diagnósticos como la depresión, el abuso de alcohol, el uso de drogas y los ataques de pánico.,14 Resnick, Acierno y Kilpatrick ${ }^{15}$ mencionan algunos mecanismos que pueden incrementar el riesgo de mayores problemas en víctimas de ataques violentos, como los son las lesiones físicas, otros problemas emocionales o la respuesta inapropiada del sistema de salud. Asimismo, el impacto subjetivo de los sucesos estresores depende de factores previos a la victimización, así como de los que ocurren durante la misma y, posteriormente, lo que muestra la complejidad de este problema.

Se ha estimado que sólo una de cuatro personas manifiesta un TPET después de un suceso traumático. ${ }^{16}$ La prevalencia anual de estrés postraumático en la población general de Estados Unidos de América alcanza $7.8 \%,{ }^{17}$ y en el ámbito mundial se ha estimado que $8 \%$ de los habitantes desarrollará este trastorno en algún momento de sus vidas. En una publicación previa a los datos de esta encuesta ${ }^{18}$ se determinó la prevalencia de estrés postraumático, según criterios de la Clasificación Estadística Internacional de Enfermedades y Problemas Relacionados con la Salud, 10a Revisión (CIE 10); $1.9 \%$ de los hombres y 3.3\% de las mujeres presentaron el diagnóstico alguna vez en su vida y $0.9 \%$ lo tenían presente en los doce meses previos al estudio. La edad promedio cuando se manifiesta el síndrome es de 19.7 años y el análisis de los hazards identificó como los momentos de mayor actividad en el reporte de las edades de inicio después de los 60 años, entre los 25 y los 35 , alrededor de los 20 años y en la infancia.

Este artículo profundiza en el análisis de la manifestación de este síndrome, reporta el índice de exposición a diferentes sucesos violentos, los correlatos demográficos, la prevalencia de estrés postraumático por tipo de evento y los efectos sobre la sociedad en la población mexicana. No se analizan en este trabajo los riesgos que la exposición a estos sucesos pueden tener en la aparición de otros trastornos como la depresión.

\section{Material y métodos}

\section{Muestra}

El estudio forma parte de la Iniciativa de la Organización Mundial de la Salud en Salud Mental, el estudio 
se lleva a cabo en forma simultánea en más de 30 países. ${ }^{19}$ La población blanco fue la no-institucionalizada, que tiene un hogar fijo, de entre 18 a 65 años de edad y que vive en áreas urbanas (población de más de 2500 habitantes). Una descripción amplia de la metodología de la Encuesta Nacional de Epidemiología Psiquiátrica realizada en México entre 2001 y 2002 ya ha sido publicada; ${ }^{18}$ brevemente, se basa en un diseño probabilístico, multietápico y estratificado para seis regiones y a escala nacional. En todos los estratos las unidades primarias de muestreo (UPM) fueron las Areas Geográficas Estadísticas Básicas (AGEB), o grupos de ellas, cartográficamente definidas por el Instituto Nacional de Estadística, Geografía e Informática (INEGI) en 1994. El tamaño promedio de las AGEB varía entre 3000 a 4000 personas de todas las edades. En total, se seleccionaron 200 UPM, distribuidas con afijación proporcional a la población de cada una de las regiones. Al interior de cada UPM se seleccionaron cinco unidades secundarias de muestreo (USM), conformadas por manzanas, o grupos de manzanas, también con probabilidad proporcional a una medida de tamaño para cada USM dentro de las UPM seleccionadas. Para cada USM seleccionada las viviendas se dividieron en segmentos compactos de alrededor de 10 de ellas. Finalmente, una sola persona se seleccionó para ser entrevistada entre los miembros de cada hogar. Las personas elegibles se definieron como los miembros del hogar que hablaran en español, que normalmente comen, duermen, preparan los alimentos y se alojan en dicho hogar, y que tenían entre 18 y 65 años de edad al momento de la entrevista.

El México urbano se estratificó en seis regiones:

1) Areas metropolitanas auto seleccionadas. Incluye las tres regiones metropolitanas más grandes del país: Ciudad de México (AMCM), Guadalajara (AMG) y Monterrey (AMM).

2) Noroeste. Incluye los estados de Baja California, Baja California Sur, Nayarit, Sinaloa y Sonora.

3) Norte. Incluye los estados de Coahuila, Chihuahua, Durango, Nuevo León (excluyendo AMM), San Luis Potosí, Tamaulipas y Zacatecas.

4) Centro-oeste. Incluye los estados de Aguascalientes, Jalisco (excluyendo AMG), Colima, Guanajuato y Michoacán.

5) Centro-este. Incluye los estados de Guerrero, Morelos, Estado de México (excluyendo AMCM), Querétaro, Hidalgo, Tlaxcala y Puebla.

6) Sureste. Incluye los estados de Veracruz, Oaxaca, Tabasco, Chiapas, Campeche, Yucatán y Quintana Roo.
Se entrevistó a 5826 individuos. La tasa de respuesta ponderada a nivel del hogar fue de $91.3 \%$, y la tasa ponderada de respuesta a nivel individual fue de $76.6 \%$.

\section{Instrumentos}

Se evaluaron 28 diferentes sucesos violentos con un instrumento cerrado, a saber, la versión computarizada del World Health Organization Composite International Diagnostic Interview (WHO-CIDI) (versión certificada 15$)^{20}$ que se instala en un computador portátil y se aplica en una entrevista cara a cara por entrevistadores legos, previamente capacitados y con controles internos que maximizan la posibilidad de una entrevista de alta calidad. ${ }^{20}$ Mediante las preguntas incluidas en el instrumento es posible obtener diagnósticos de acuerdo con el DSM IV y CIE 10. En este artículo se presentan los diagnósticos de acuerdo con El DSM IV. El WHO-CIDI contiene además módulos sobre las características sociodemográficas de los entrevistados, condiciones crónicas, fármaco epidemiología, discapacidad y uso de servicios. La confiabilidad y validez ha sido ampliamente documentada. La traducción de la encuesta al español fue hecha conforme con las recomendaciones de la OMS.

Con el fin de maximizar la obtención de información al tiempo que minimizar el tiempo de entrevista, algunos individuos de la muestra contestaron la versión larga del instrumento y otros la resumida, seleccionados con base en sus respuestas a las preguntas de tamizaje (preguntas que indican probabilidad de que el diagnóstico esté presente), y por selección aleatoria con probabilidad proporcional al número de sujetos que habitaban las viviendas de la muestra. El tiempo máximo de entrevista fue de nueve horas en cuatro sesiones y el mínimo de 20 minutos.

La sección sobre TEPT contiene 281 preguntas. Está formada por una primera sección de tamizaje en la que se pregunta a los entrevistados la exposición a 28 diferentes sucesos (ver anexo); para cada uno de estos sucesos, se pregunta a continuación: la edad a la que ocurrió el evento, el tiempo que estuvo el sujeto expuesto al evento, el número de veces que le ocurrió el evento, los síntomas asociados al evento, la duración de los síntomas; en caso de que el sujeto haya tenido varios sucesos se le pregunta, además, cuál fue el peor de éstos y cuáles fueron los síntomas que lo acompañaron. Como ya se mencionó, el diagnóstico del trastorno se derivó de los criterios establecidos por el DSM-IV: rexperimentación del suceso, evitación y síntomas de hiperactivación fisiológica. 
Las mediciones de discapacidad empleadas fueron: la escala de discapacidad de Sheehan ${ }^{22}$ y el autoreporte del entrevistado sobre el número de días durante los cuales fue totalmente incapaz de realizar sus actividades. La escala de discapacidad de Sheehan (1983) fue diseñada para evaluar las limitaciones que los trastornos mentales producen en el funcionamiento de las personas. Originalmente constaba de tres preguntas que evaluaban el impacto que los síntomas habían tenido sobre el trabajo, la vida social y la vida familiar/hogar del paciente. En esta investigación, el reactivo correspondiente a la vida familiar se desglosó en dos: uno, relacionado con las actividades del hogar y el otro, con las relaciones con personas cercanas.

La evaluación se hace sobre una línea horizontal con marcas numéricas y verbales en respuesta a la pregunta: ¿qué número describe mejor en qué medida sus reacciones debido al suceso sobre el que se interroga, (el peor suceso de los 12 meses/esos sucesos) dificultaron cada una de las siguientes actividades?, (el trabajo, la vida social, las actividades del hogar y las relaciones con personas cercanas). La escala de respuestas es de 0 a 10. El 0 se acompaña del descriptor verbal "ninguna dificultad", del 1al 3 corresponden a "leve", 4 a 6 "moderada", 7 a 9 "grave" y 10 "dificultad muy grave". La consistencia de los descriptores verbales ya ha sido referida.*

La otra medida de discapacidad fue la respuesta del sujeto a la pregunta: aproximadamente, ¿cuántos días de los últimos 12 meses fue totalmente incapaz de trabajar o hacer sus actividades habituales debido a sus reacciones ante (el peor suceso de los 12 meses/ esos sucesos)? Se presentan los promedios de los pacientes que cumplieron los criterios para el diagnóstico de TEPT en cada una de las cuatro áreas mencionadas.

El estudio de discapacidad muestra un interés mayor a partir de la aparición de la Clasificación Internacional de Funcionamiento de la Organización Mundial de la Salud que evalúa la calidad de vida, dimensión complementaria a la de enfermedad, contenida en la CIE; incorpora los conceptos de daño en el organismo, las limitaciones en el funcionamiento que resultan de la lesión o daño y las limitaciones en la participación social que se derivan de las condiciones sociales en las que se desarrolla la persona y que pueden variar de un escenario a otro, dependiendo de fac-

\footnotetext{
* Lara C, Medina-Mora ME, Borges G, Zambrano J. Trastornos mentales y discapacidad. El costo social de la enfermedad mental. Enviado a publicación.
}

tores como la disponibilidad de transporte, habitación adaptada, y del estigma y discriminación. Al incorporar este segundo eje en la evaluación de la carga de la enfermedad, las enfermedades mentales cobraron importancia pasando del $12^{\circ}$ lugar, cuando se clasificaban en función de la mortalidad asociada, al $5^{\circ}$ lugar, cuando se define la carga por mortalidad prematura y días de vida sanos vividos con discapacidad. ${ }^{23}$ Las enfermedades mentales, salvo algunas excepciones como es el caso del suicidio asociado con mayor frecuencia a la depresión, no causan muerte directa, pero los enfermos tienden a vivir una vida con alto nivel de discapacidad.

\section{Procedimiento}

El trabajo de campo fue llevado a cabo por 34 entrevistadores con amplia experiencia en el levantamiento de encuestas. Recibieron una capacitación inicial de cinco días, proporcionada por profesionales certificados por la OMS. Se diseñó una serie de actividades, con el fin de asegurar el control de calidad de la información.

Es importante mencionar que a todos los encuestados se les presentó una carta explicando el proyecto, en la cual se les informaba que todos los datos que se recabaran serían tratados como confidenciales y, a su vez, se les proporcionaron distintos números telefónicos para verificar que se estaba llevando a cabo la encuesta y aclarar cualquier duda al respecto. Debido a que las entrevistas se realizaron con computadora portátil, se les leyeron a los sujetos dos escritos que aparecían en la pantalla: en el primero se les recordó que su participación era voluntaria y confidencial, así como su derecho a no responder las preguntas que no desearan. En el segundo escrito se les pidió su consentimiento y en caso de que no lo proporcionaran el programa de cómputo no permitía seguir levantando la entrevista. Al terminar la entrevista se les entregó a los encuestados una lista de las instituciones de salud a donde podían acudir si deseaban hablar más sobre los temas tratados.

El protocolo de la investigación fue aprobado por el Comité de Etica del Instituto Nacional de Psiquiatría Ramón de la Fuente el día 4 de julio de 2001.

\section{A nálisis de los datos}

Los errores estándar de las proporciones (prevalencia de por vida, 12 meses y 30 días) se obtuvieron por medio del método de linearización de Taylor utilizando el paquete estadístico SUDAAN. ${ }^{24}$ Se utilizó el método de Kaplan-Meir ${ }^{25}$ para generar las curvas de edades de inicio y de sobrevida usando el paquete SAS. 
Se realizaron análisis de regresión logística ${ }^{26}$ para estudiar los correlatos demográficos del estrés postraumático. Las estimaciones de los errores estándar de las razones de momios (Odds Ratio, OR) para los coeficientes de la regresión logística fueron obtenidos también mediante el SUDAAN, con intervalos del coeficiente de $95 \%$ ajustados al efecto del diseño.

\section{Resultados}

Alrededor de $54 \%$ de la muestra fue del sexo femenino, y alrededor de $40 \%$ se ubicó en el grupo de edad más joven (18 a 29 años). Casi $68 \%$ de la población contó únicamente con estudios primarios (sexto grado), y sólo $12 \%$ completó estudios universitarios. La mayoría reconoció como estado civil el estar casado o en unión libre (67\%), y estar trabajando actualmente (58\%) (cuadro I).

¿Qué tan expuestos están hombres y mujeres a la violencia?

A los individuos de la muestra se les preguntó por cada uno de los 28 sucesos que conforman la sección de estrés postraumático; la descripción de esta parte se incluye en el anexo. Los resultados del estudio mostraron que $68 \%$ de la población adulta que vive en el México urbano ha estado expuesta al menos a un suceso estresante alguna vez en su vida. El 20\% dijo haber experimentado al menos uno de los sucesos, $15 \%$ dos y $33 \%$ tres o más.

Las prevalencias de los 28 sucesos susceptibles de generar un TEPT se muestran en el cuadro II, considerando, además, su distribución por sexo y si existieron diferencias estadísticamente significativas en términos de su presencia en hombres y mujeres. Como se observa, una de cada cuatro personas había experimentado la muerte repentina o inesperada de un familiar o amigo cercano (26.9\%), así como algún atraco en el que fue amenazada con un arma $(24.6 \%)$. Una de cada cinco $(21.4 \%)$ ha tenido algún accidente; en proporción similar la población ha sido testigo de violencia doméstica en su infancia (20.3\%) y víctima de violencia física severa (apaleado) por parte de padres o cuidadores en la infancia (18.3\%).

El 16.3\% ha sido testigo de la muerte o lesiones de una persona, $13.7 \%$ experimentó algún desastre natural o provocado por el hombre. Una de cada 10 personas ha sufrido alguna enfermedad grave; $7.4 \%$ ha sido apaleado por alguna persona que no son los padres $\mathrm{o}$ la pareja, $6 \%$ ha sido apaleado por su pareja y $5.4 \%$ reporta haber experimentado abuso sexual. Prevalencias menores, pero importantes de mencionar, son la violación con 3.9\% y la persecución o acoso con 3.7\%.

\section{Cuadro I \\ Datos sociodemográficos. Encuesta Nacional de Epidemiología Psiquiátrica, México, 2001-2002}

Población mexicana \% $\quad n$

Sexo

\begin{tabular}{lll} 
Hombres & 47.71 & 1127 \\
\hline Mujeres & 52.29 & 1235
\end{tabular}

Edad (años)

\begin{tabular}{rrr}
$18-29$ & 41.29 & 975 \\
\hline $30-44$ & 34.57 & 816 \\
\hline $45-59$ & 18.91 & 447 \\
\hline $60-65$ & 5.23 & 124
\end{tabular}

Estado civil

\begin{tabular}{lrr} 
Soltero & 25.63 & 605 \\
\hline Casado/unión libre & 67.32 & 1590 \\
\hline Separado/viudo/divorciado & 7.06 & 167
\end{tabular}

$N$ ivel educativo

\begin{tabular}{lrr} 
Primaria & 68.53 & 1616 \\
\hline Secundaria & 12.79 & 301 \\
\hline Preparatoria & 6.76 & 159 \\
\hline Licenciatura & 11.93 & 281
\end{tabular}

Situación laboral

\begin{tabular}{lrr} 
Trabaja actualmente & 58.36 & 1379 \\
\hline Estudia & 6.91 & 163 \\
\hline Hogar & 28.80 & 680 \\
\hline Jubilado & 1.46 & 34 \\
\hline O tro & 4.47 & 106
\end{tabular}

Región

\begin{tabular}{lrl} 
Areas metropolitanas & 27.62 & 653 \\
\hline N oroeste & 8.02 & 189 \\
\hline N orte & 15.15 & 358 \\
\hline Centro oeste & 12.42 & 293 \\
\hline Centro este & 17.52 & 414 \\
\hline Sureste & 19.27 & 455
\end{tabular}

Cabe señalar que solamente $1.4 \%$ de los entrevistados reportó algún otro suceso traumático diferente a los de la lista del estudio.

En cuanto a las diferencias por sexo, destaca que las mujeres presentan prevalencias estadísticamente ( $£ 0.05)$ más elevadas de abuso sexual, violencia física por parte de la pareja, violación, persecución y acoso. Por su parte, los hombres experimentaron más atracos con arma, así como accidentes; también fueron testi- 


\section{Cuadro II \\ Prevalencias de los sucesos, por sexo. Encuesta Nacional de Epidemiología Psiquiátrica, Méxıco, 2001-2002}

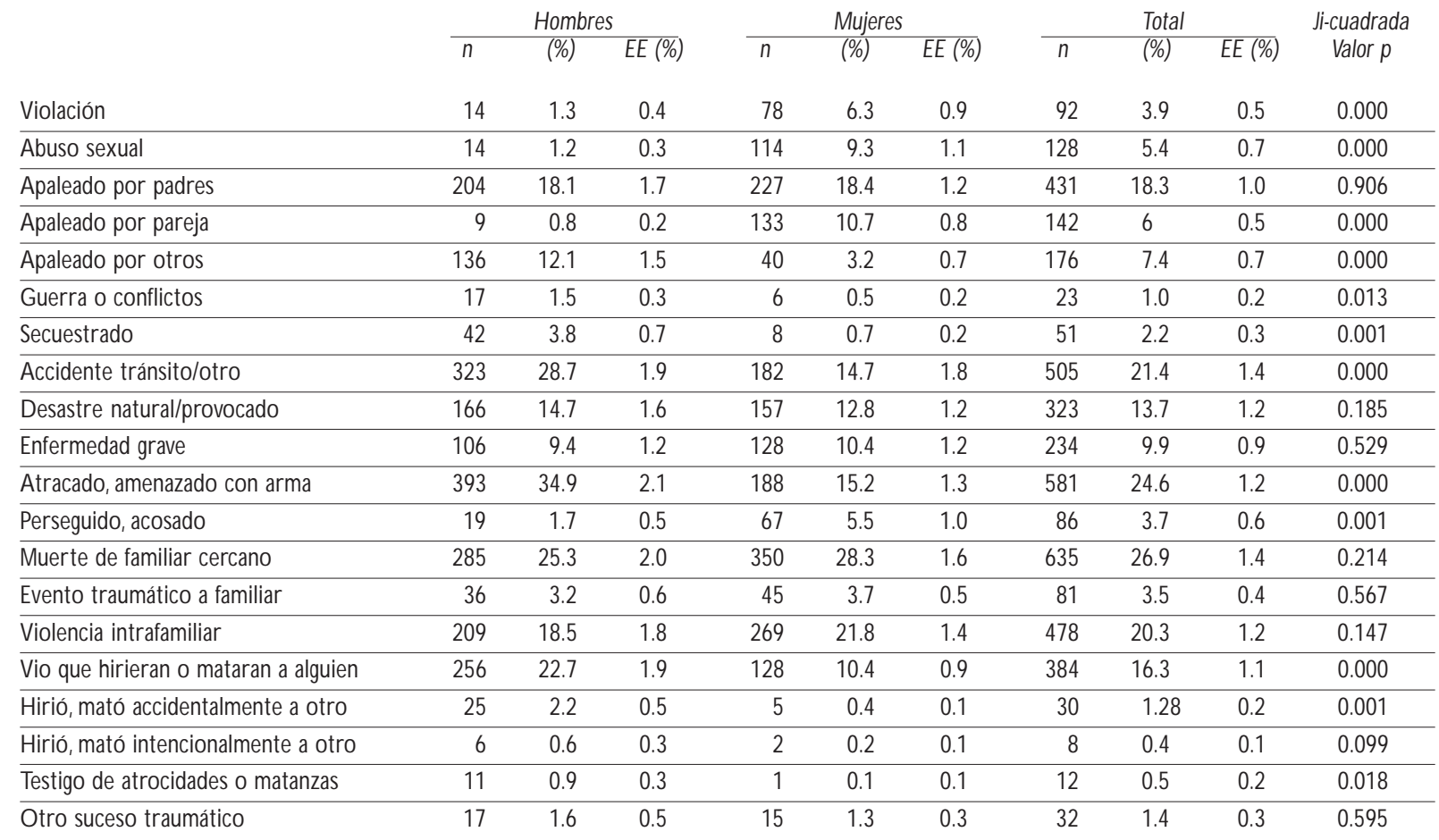

$\mathrm{EE}=$ error estándar

gos, en mayor proporción que las mujeres, de muertes o de lesiones, sufrieron más secuestros, mataron accidentalmente a otro en un porcentaje mayor, participaron en guerras o conflictos y fueron testigos de atrocidades o matanzas.

¿En qué regiones de la república han ocurrido estos sucesos?

El cuadro III muestra la distribución regional de los diferentes sucesos, siguiendo la regionalización que sirvió de base para la selección de la muestra. Tres sucesos mostraron una distribución significativamente diferente entre las regiones del país: el secuestro, el atraco o amenaza con armas y la experiencia de haber sido testigo de que hirieran o mataran a alguien. El secuestro en la región noroccidental del país es hasta seis veces más frecuente que en el sur, 3.3 veces más frecuente de lo que ocurre en las tres grandes zonas metropolitanas, (el Distrito Federal, Monterrey y Guadalajara). El atraco y las amenazas con armas ocurren significativamente con mayor frecuencia en las zonas metropolitanas, 33\% de los habitantes de estas zonas dijeron haber tenido una experiencia de este tipo, en comparación con sólo 17\% en el sureste. La región centro oeste ocupo el primer lugar en el ser testigo de la muerte de otra persona o el que hubiera resultado herida (26.4\%); las zonas metropolitanas ocupan el segundo lugar $(19.4 \%)$.

¿En qué etapa del desarrollo se está más expuesto a la violencia?

Las figuras 1 y 2 muestran las curvas de sobrevida que señalan las edades de inicio o de la primera exposición para diferentes tipos de sucesos. Como puede verse, existen grandes variaciones. Por ejemplo, el haber causado lesiones a otros ocurre con más frecuencia en la adolescencia, mientras que el sufrir la muerte de un familiar cercano ocurre a lo largo de la vida, con pocas diferencias en los estadios de desarrollo.

La experiencia de acoso, tiene su inicio alrededor de los 15 años de edad, siendo más frecuente antes de los 30 y ocurre en menos proporción hasta los 40; los 


\section{Cuadro III}

\section{Distribución Regional de la pReVAlencia de suCesos estresantes en la República mexicAna.} Encuesta Nacional de Epidemiología Psiquiátrica, México, 2001-2002

\begin{tabular}{|c|c|c|c|c|c|c|c|c|c|c|}
\hline \multirow[b]{3}{*}{ Región } & \multicolumn{2}{|c|}{ Eventos accidentales } & \multicolumn{2}{|c|}{ Violencia } & \multicolumn{2}{|c|}{ Violencia familiar } & \multirow{3}{*}{ Secuestro } & \multirow{3}{*}{ Atraco } & \multirow{3}{*}{$\begin{array}{c}\text { Violencia social } \\
\text { Evento } \\
\text { traumático } \\
\text { a familiar cercano } \\
\%\end{array}$} & \multirow{3}{*}{$\begin{array}{c}\text { Vio que hirieran } \\
\text { o mataran } \\
\text { a otro } \\
\%\end{array}$} \\
\hline & Accidente & $\begin{array}{c}\text { Muerte } \\
\text { de familiar }\end{array}$ & Sexual & $\begin{array}{l}\text { Cónyuge } \\
\text { Otros }\end{array}$ & Padres & $\begin{array}{l}\text { Intra- } \\
\text { familiar }\end{array}$ & & & & \\
\hline & $\%$ & cercano & $\%$ & & $\%$ & $\%$ & & & & \\
\hline & & & 01 & 120 & $10 ?$ & 100 & 10 & & 17 & 191 \\
\hline Noroeste & 19.2 & 25.4 & 7.2 & 14.7 & 24.6 & 12.3 & 1.4 & 20.9 & 3.5 & 6.6 \\
\hline Norte & 26.0 & 35.5 & 6.0 & 14.9 & 17.2 & 16.1 & 6.3 & 21.2 & 1.6 & 14.6 \\
\hline Centro-oeste & 27.3 & 33.2 & 9.3 & 14.6 & 20.9 & 18.1 & 0.7 & 22.9 & 4.5 & 26.4 \\
\hline Centro-este & 20.1 & 20.4 & 7.1 & 15.1 & 18.1 & 26.2 & 1.8 & 24.5 & 3.6 & 11.5 \\
\hline Sureste & 17.0 & 26.7 & 6.2 & 8.6 & 15.1 & 23.3 & 1.0 & 17.6 & 2.3 & 15.1 \\
\hline$\chi^{2}$ (valor $p$ ) & 0.400 & 0.081 & 0.510 & 0.262 & 0.316 & 0.132 & 0.010 & 0.001 & 0.094 & 0.000 \\
\hline
\end{tabular}

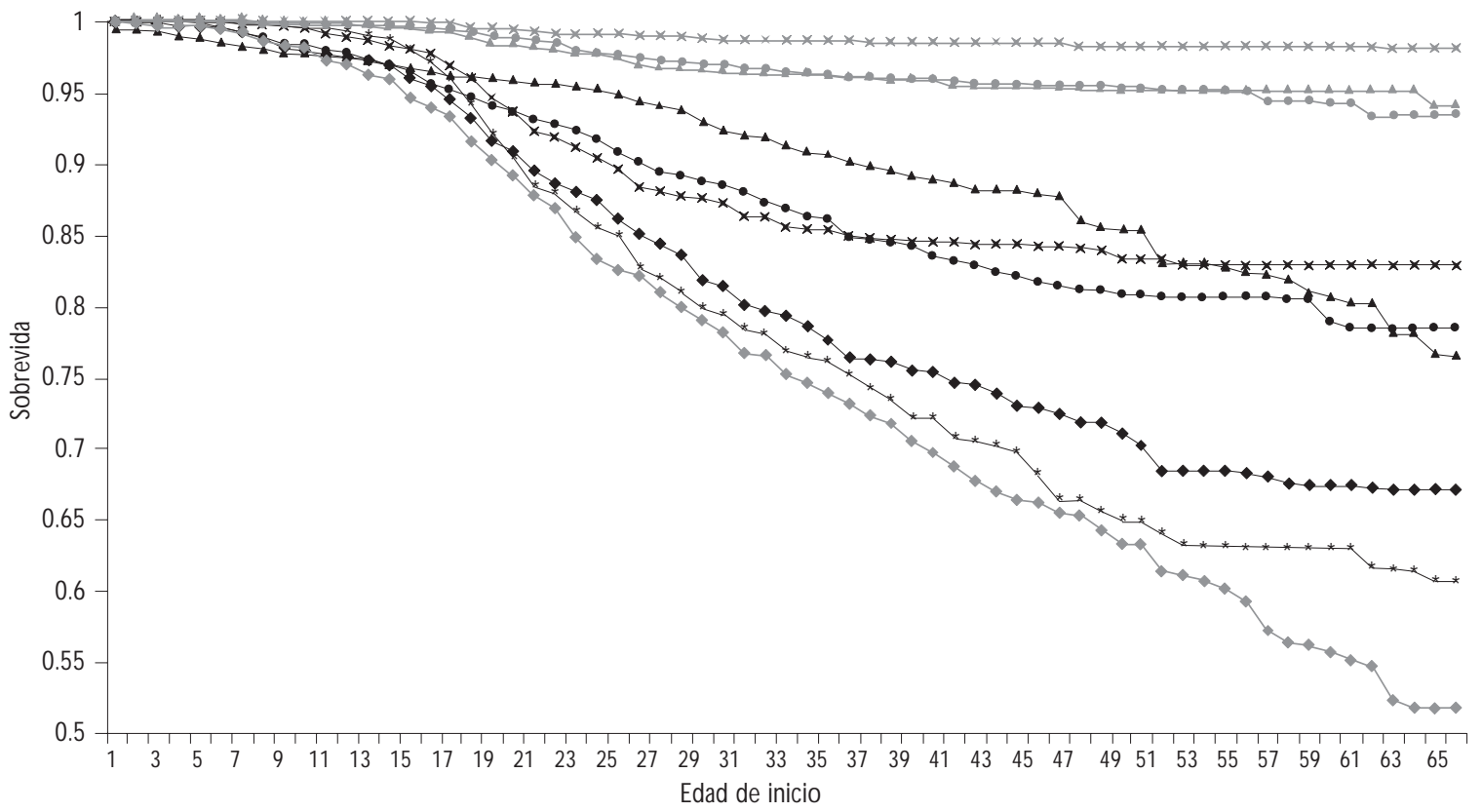

\footnotetext{
- Accidente grave

- Desastre natural o provocado

- Enfermedad casi fatal
}
* A paleado por otros (no padres)
* Atracado, amenazado por arma
^- Perseguido, acosado

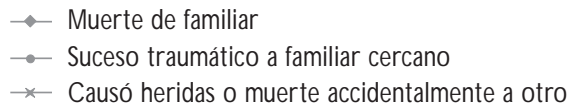

Figura 1. Edad de inicio para diferentes sucesos traumáticos. Encuesta Nacional de Epidemiología PsiquiáTRICA, MÉXICO, 2001-2002

nuevos casos son raros después de esta edad y vuelven a tener un incremento al pasar los 60. Los accidentes graves se expresan en forma más evidente en la adolescencia, los nuevos casos tienen un rápido crecimiento en la juventud y empiezan con un crecimiento menos acelerado después de los 40, y lento después de los 50 .

Los atracos o amenazas con armas ocurren en todas las edades, aunque empiezan a ser más frecuentes en 


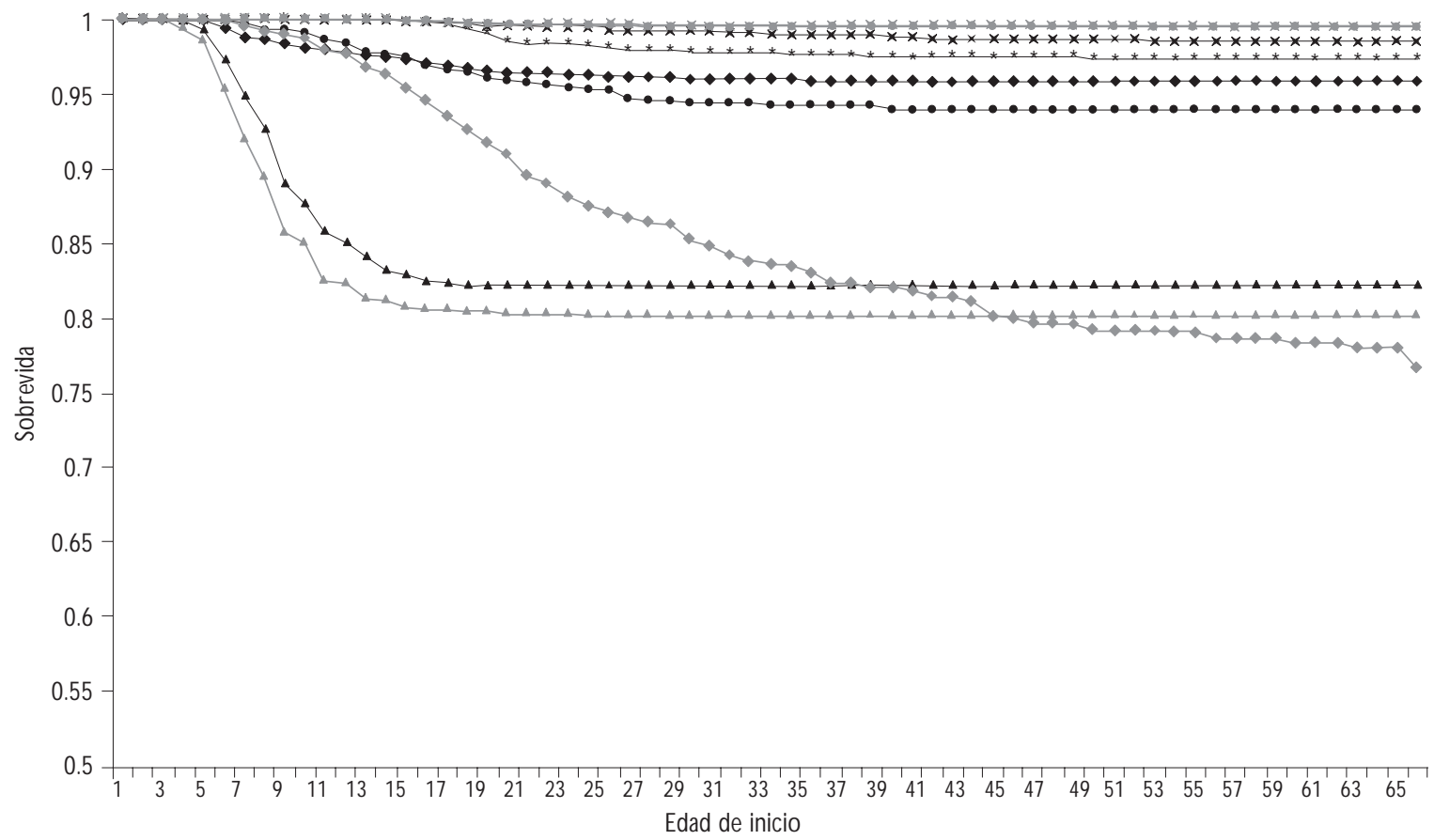

—- Violación sexual
- Abuso sexual
- A paleado por padres

Figura 2. Edad de inicio para diferentes sucesos traumáticos. Encuesta Nacional de Epidemiología PsiquiáTRICA, MÉXICO, 2001-2002 la adolescencia, se manifiestan en forma continua hasta los 40 años, y son poco frecuentes en las dos siguientes décadas hasta los 60 años, edad cuando la población que no había experimentado estos sucesos antes, vuelve a estar en riesgo.

En la figura 2 se muestra un segundo grupo de sucesos traumáticos que con frecuencia permanecen en secreto y ocurren en ámbitos privados. El evento que tiene una aparición más temprana es el haber sido testigo de violencia doméstica, es decir, haber visto peleas físicas, en especial del padre y de la madre, con una frecuencia amplia entre los 5 y los 10 años de edad, con menor incidencia entre los 10 y los 20 años y con poco incremento en el número de nuevos casos a partir de esta edad, lo que de alguna manera se explica por la propia pregunta que limita el tiempo de exposición al suceso a la infancia. El ser víctima de violencia por parte de los padres mostró una distribución similar, pero los nuevos casos dejan de aparecer a una edad más temprana, siendo poco probable que ocurran después de los 17 años. Como se puede observar al comparar las figuras 1 y 2 algunos sucesos están muy limitados en su edad de inicio y si no sucede temprano en la infancia, es muy poco probable que suceda en etapas posteriores (como la violencia intrafamiliar o ser apaleado por los padres). En contraste, los sucesos como la muerte de un familiar pueden suceder en cualquier momento de la vida del entrevistado.

Los niños y los adolescentes están más expuestos a la violación sexual; este evento tiene su aparición en las dos primeras décadas de la vida, con pocos nuevos casos después de los 20 años. El abuso sexual ocurre también por primera vez en edades tempranas con reportes a partir de los cinco años de edad; el índice de nuevos casos sigue apareciendo hasta los 25 años, con un segundo período con menos casos nuevos hasta poco después de los 30 años, y con poca incidencia después de esta edad.

¿Cuáles son los sucesos asociados con una mayor prevalencia de trastorno por estrés postraumático?

La prevalencia total de trastorno por estrés postraumático fue de $1.45 \% ; 2.3 \%$ en las mujeres y $0.49 \%$ en

salud pública de méxico / vol.47, no.1, enero-febrero de 2005 
los hombres. Como se observa en el cuadro IV, las prevalencias más elevadas según el criterio DSM-IV, fueron las asociadas a la persecución o acoso, la violación, el secuestro, el abuso sexual y el haber sido víctima de violencia por parte de los padres.

\section{¿Cuál es el impacto social de este trastorno?}

Con el fin de evaluar el impacto social del estrés postraumático se pidió a la población que indicara, en una escala de 0 a 10, el grado de dificultad en la vida diaria en el hogar, el trabajo, las relaciones interpersonales y la vida social. Las personas que tuvieron el diagnóstico el año anterior al estudio ( $0.6 \%$ de la población) reportaron en promedio un puntaje de dificultad entre moderado y severo, con problemas de funcionamiento en todas las áreas de la vida. En una escala de 0 a 10, las personas con estrés postraumático comunicaron un índice de dificultad moderada, con un promedio de 5.79 de dificultad para llevar a cabo las actividades del hogar, 5.88 actividades de la vida social, 5.93 para las relacionadas con el trabajo y, en el nivel más alto, 6.07, la dificultad para relacionarse con personas cercanas.

También se preguntó a la población con diagnóstico de TEPT en el año previo al estudio cuántos días había sido totalmente incapaz de trabajar o hacer sus

\section{Cuadro IV \\ Prevalencia de trastorno por estrés postraumático, según sucesos. Encuesta Nacional de Epidemiología Psiquiátrica, México, 2001-2002}

\begin{tabular}{lrrr} 
& $n$ & TEPT (\%) & EE (\%) \\
Violación & 10 & 11.3 & 3.0 \\
\hline A buso sexual & 7 & 5.6 & 1.8 \\
\hline A paleado por padres & 18 & 4.3 & 1.1 \\
\hline Secuestrado & 3 & 5.6 & 2.4 \\
\hline Accidente tránsito/otro & 17 & 3.5 & 1.0 \\
\hline Desastre natural/provocado & 7 & 2.3 & 1.2 \\
\hline Enfermedad grave & 8 & 3.5 & 1.1 \\
\hline A paleado por otros (no padres) & 11 & 3.6 & 0.9 \\
\hline Atracado, amenazado con arma & 10 & 1.8 & 0.5 \\
\hline Perseguido, acosado & 7 & 8.1 & 4.4 \\
\hline Muerte de familiar cercano & 25 & 4.0 & 0.9 \\
\hline Violencia intrafamiliar & 14 & 3.1 & 1.0 \\
\hline Vio que hirieran o mataran a alguien & 9 & 2.6 & 0.8 \\
\hline O tro suceso traumático & 5 & 3.7 & 1.0
\end{tabular}

TEPT $=$ trastorno por estrés postraumático $\mathrm{EE}=$ error estándar

salud pública de méxico / vol.47, no.1, enero-febrero de 2005 actividades habituales debido al trastorno. El total de días con discapacidad en el último año fue de 21.4 por persona. Si consideramos el salario promedio de la población, que alcanzó $\$ 64.00^{27}$ podríamos estimar la pérdida en un año en $\$ 323000000.00$.

\section{Discusión}

Las encuestas son una metodología de gran utilidad para conocer y caracterizar sucesos estresantes; asimismo, permiten hacer visibles expresiones de violencia que no son reportadas al sistema de justicia, al de salud o a otras instancias, en gran medida por no conllevar necesariamente lesiones físicas severas. ${ }^{4} \mathrm{Sin}$ embargo, no dejan de tener limitaciones.

En el caso de sucesos violentos, esta situación es particularmente difícil debido a que: a) por mecanismos defensivos de corte evitativo, hay sucesos traumáticos que pueden no recordarse o sobre los que puede negarse su importancia,; ${ }^{28}$ recordemos también que si ocurrieron en edades tempranas o han sido repetidos y de larga duración, hay una alta probabilidad de que sean recordados en forma confusa o distorsionada; ${ }^{29} \mathrm{~b}$ ) también puede ocurrir que no se admita haber experimentado ciertos hechos violentos porque generan vergüenza o temor, como sucede con los de tipo sexual o familiar, y c) en otros casos, la información puede ocultarse debido a que los agresores pueden vivir en el mismo domicilio de los entrevistados, por lo que es preferible que ésta se mantenga en secreto por el peligro al que se expone a la persona que denuncia los sucesos violentos al ser agredida en forma severa.

En cuanto al primer punto, cabe señalar que es imposible tener un control sobre este tipo de distorsión cognitiva, sin embargo, consideramos que es una situación que se distribuye en forma similar en la población. Respecto al segundo, es decir, la vergüenza o temor a reportar alguna victimización, en este estudio se preguntó a las personas si había algún otro evento violento que le hubiera ocurrido, pero que prefería mantener en secreto; en caso positivo, se hacían todas las preguntas sobre este evento secreto. De esta manera, aunque no sepamos exactamente qué suceso ocurrió, es posible calcular su impacto en caso de haberlo sufrido. Asimismo, tomando como base las sugerencias del documento "Dando prioridad a las mujeres: recomendaciones éticas y de seguridad para la investigación sobre la violencia doméstica contra las mujeres", ${ }^{30}$ también se salvaguardó la seguridad de los entrevistadores, ya que cada grupo de ocho encuestadores estaba acompañado de dos supervisores, quienes sabían en qué hogares estaban llevándose a cabo 
entrevistas y estaban pendientes del tiempo y condiciones de la entrevista. Asimismo, a los sujetos entrevistados se les ofrecían opciones de ayuda en su municipio, mismas a las que podían acudir en caso de así desearlo. Cabe destacar que el considerar los aspectos anteriores permitió, además, obtener una información de alta calidad.

Los resultados del estudio nos indican una alta exposición en la población nacional a sucesos susceptibles de generar un estado traumático, siendo los más frecuentes la muerte de un familiar cercano, el haber sido víctima de un robo o la amenaza con arma y los accidentes. Los menos frecuentes fueron la experiencia de guerra, el haber sido testigo de matanzas, y el homicidio imprudencial o intencional.

En aquellos sucesos que ocurren con poca participción o control humano, como los desastres naturales o las enfermedades graves, no se observan diferencias entre los sexos. Lo mismo ocurre con el haber sido víctima o testigo de violencia por parte de los padres en la infancia, y el haber experimentado violencias vicariamente (sucesos traumáticos a familiares o amistades).

En concordancia con la literatura internacional, los hombres están más expuestos a sucesos como los accidentes y violencias que ocurren en espacios públicos como guerra, secuestro, y asaltos o a haber sido testigo de lesiones, homicidios y matanzas, así como el haber herido o matado a otra persona accidentalmente. Este tipo de situaciones se derivan en gran medida de su condición de género, específicamente de comportamientos asociados con una construcción de la masculinidad "riesgosa para la salud" ${ }^{31}$ y un papel masculino tradicional en el que destaca el involucrase en actividades de alto riesgo. ${ }^{32}$

Las mujeres, por su parte, están más expuestas a violencias también derivadas de su condición de género, tales como la sexual y la violencia por parte de la pareja. Los resultados de esta encuesta coinciden con los de la Encuesta Nacional sobre Violencia contra las Mujeres $2003^{8}$ efectuada en servicios de salud, en la que se reporta un índice de $9.8 \%$ de violencia física; en este estudio se detectó $10.7 \%$. Asimismo, el que también se encontrara una proporción mayor de mujeres que habían sufrido persecución o acoso, pone en la mesa un tema importante que ha recibido poca atención, pues desconocemos si este acoso se relaciona también con la condición de género.

Los datos parecen mostrar que efectivamente las mujeres tienden a experimentar ciertas formas de violencia por el hecho de ser mujeres, lo que incluye a "todo acto de fuerza física o verbal, coerción o privación amenazadora para la vida, dirigida al individuo mujer o niña, que cause daño físico o psicológico, humillación o privación arbitraria de la libertad y que perpetúe la subordinación femenina". ${ }^{33}$ Además, como se analizará más adelante, los sucesos que les ocurren a las mujeres se asocian con secuelas más severas, como lo muestran los porcentajes más elevados de trastorno por estrés postraumático, tal y como lo revela la literatura revisada internacionalmente. ${ }^{4,16}$

Desafortunadamente, la encuesta muestra que muchos sucesos susceptibles de generar un trauma ocurren en la infancia y en la adolescencia. Los eventos que tiene una manifestación más temprana son la violencia intrafamiliar, y los golpes de los padres. Los niños y los adolescentes están más expuestos a la violación sexual; este evento tiene su aparición en las dos primeras décadas de la vida, con pocos nuevos casos después de los 20 años. El abuso sexual ocurre también por primera vez en edades tempranas con reportes a partir de los cinco años de edad; el índice de nuevos casos sigue apareciendo hasta los 25 años. Esto manifiesta la importancia de dirigir la atención a la prevención de la violencia en edades tempranas, así como del tratamiento de sus secuelas, ya que precisamente la ocurrencia de situaciones traumáticas en estas edades parece asociarse con problemas psiquiátricos más severos a lo largo de la vida. ${ }^{34}$ Asimismo, al explorar a través de nuevas técnicas, como la formación de imágenes que registran la actividad cerebral, los maltratos inferidos durante la infancia, se ha observado que pueden causar un daño permanente en la estructura neural y en el funcionamiento de un cerebro que apenas se está desarrollando. ${ }^{35}$ Desafortunadamente, estos sucesos violentos son, además, ejercidos principalmente por las personas que supuestamente son las encargadas del cuidado y el apoyo afectivo de los infantes.

En el caso de la adolescencia, el haber causado lesiones a otros ocurre, por primera vez, con más frecuencia en esta etapa del desarrollo; la experiencia de acoso tiene su inicio alrededor de los 15 años de edad y vuelve a tener un incremento posteriormente al pasar los 60 años, y los accidentes graves se expresan en forma más evidente en la adolescencia. Los atracos o amenazas con armas aunque ocurren en todas las edades, son más frecuentes en la adolescencia, se manifiestan en forma continua hasta los 40 años, y es en la tercera edad cuando la población vuelve a estar en riesgo. Como se hace evidente, los sucesos violentos que empiezan a ocurrir en esta fase de la vida son cualitativamente diferentes a los anteriores, pues implican el ejercicio de la violencia y el experimentar diferentes sucesos que, al menos en el caso de los accidentes graves y los atracos y amenazas con armas, parecen ocurrir en contextos públicos, más que privados. El caso del acoso, como 
ya se comentó, es un problema que requiere profundizarse debido a que, además, fue mucho más frecuente en las mujeres.

Los datos de la encuesta muestran importantes variaciones regionales en la exposición a la violencia; así, el secuestro ocurre con más frecuencia en la región noroccidental del país en donde es hasta seis veces más frecuente que en el sur del país. Coincidente con los datos de Encuesta Nacional sobre Inseguridad (ENSI-1), ${ }^{5}$ el atraco y amenazas con armas ocurren significativamente más frecuentemente en las zonas metropolitanas. La región centro oeste ocupó el primer lugar en el ser testigo de la muerte de otra persona o el que hubiera resultado herida, las zonas metropolitana ocuparon el segundo lugar.

En total, $1.46 \%$ de la población ha experimentado estrés postraumático; según las definiciones del DSM IV, este índice es inferior al reportado en EUA de 7.8\% usando la clasificación previa del DSM IIIR. ${ }^{36}$ El análisis de los correlatos demográficos ha permitido mostrar como variables fundamentales el sexo, la edad y la escolaridad. Estudios internacionales muestran que las mujeres suelen reportar en porcentajes más elevados el trastorno, que los hombres, ${ }^{17}$ por lo general presentan el doble de posibilidades de desarrollar TEPT. ${ }^{16}$ El impacto sobre la vida de las personas tiene que ver con todas las áreas de su vida social y sobre todo, con su visión del mundo y de sí mismas.

En esta encuesta, se confirman las marcadas diferencias por sexo. A pesar de que hombres y mujeres estuvieron expuestos en forma importante a la violencia, la proporción de mujeres que desarrollan el trastorno es considerablemente mayor, 4.73 mujeres por cada hombre. Esto puede estar relacionado con el estigma social que acompaña a sucesos tales como el abuso sexual o la violación, que una vez ocurridos tienen repercusiones de por vida y a que son los más frecuentes entre las mujeres.

Si consideramos los tipos de sucesos, se reporta que este trastorno es una reacción más frecuente en víctimas de delitos violentos y, en particular, en víctimas de violencia sexual, abuso sexual o maltrato físico severo en la infancia ${ }^{4,17,37}$ y en mujeres víctimas del crimen, especialmente violación y tortura, y en sobrevivientes de campos de concentración. ${ }^{38}$ En este estudio el TEPT efectivamente fue más elevado en personas expuestas a la violación y al acoso o la persecución; siendo también muy impactantes el secuestro y el abuso sexual, lo que nos habla de la importancia de profundizar en el estudio de estos problemas, en términos de sus características, severidad, temporalidad, recurrencia y cronicidad. La violencia sexual ha mostrado en otros estudios que efectivamente se asocia con una gran cantidad de síntomas, incluyendo aquellos rela- cionados con el estrés postraumático que persisten aún cuando hayan pasado muchos años desde que se sufrió el abuso. ${ }^{39}$ Asimismo, que los problemas de salud mental como la depresión, el intento suicida y el uso de drogas se relacionan también con este tipo de violencia. ${ }^{40-43}$ Sin embargo, tenemos mucho menos información sobre el secuestro, la persecución y el acoso, lo que muestra la importancia de desarrollar líneas de investigación al respecto.

Por otro lado, análisis previos de la encuesta han mostrado un bajo índice de utilización de servicios en la población afectada ${ }_{1}^{18}$ menor de lo que se reporta para EUA, si bien la prevalencia de trastornos de la ansiedad, entre los que se incluye el trastorno por estrés postraumático, es 2.6 veces inferior en México $(6.8 \%$ y $18.2 \%$, prevalencia en 12 meses previos a la encuesta), la proporción de enfermos que recibe tratamiento es 3.6 veces inferior en México, en comparación con lo que ocurre en EUA (4.3\% y $15.3 \%$, respectivamente). ${ }^{44}$

En cuanto al costo social derivado de este desorden, las personas con estrés postraumático presentan dificultad, de moderada a severa, en todas las esferas de la vida, con importantes pérdidas económicas. Las dificultades en el funcionamiento son elevadas, y en general, más elevadas aún cuando se sufre estrés postraumático en comparación con lo que se observa en otros fenómenos.

\section{Conclusiones}

Los resultados muestran un índice importante de victimización, con importantes variaciones entre hombres y mujeres; las segundas están expuestas con más frecuencia a sucesos relacionados con la "violencia de género", asociados con un mayor riesgo de desarrollar un síndrome de estrés postraumático. Los hombres muestran, por su parte, mayor exposición a sucesos relacionados con su presencia en los espacios públicos y con comportamientos que pueden también ser parte de comportamientos "esperados" en los hombres, y que los ponen en riesgos de ejercer o experimentar violencia.

Los sucesos, además, ocurren de manera diferente de acuerdo con el estadio de desarrollo psicosocial del individuo, teniendo mayor riesgo en la infancia, la adolescencia y la tercera edad. La exposición a la violencia por parte de familiares y la de tipo sexual es altamente frecuente en las edades tempranas. De esta manera, se distinguen claramente los grupos vulnerables a la violencia, tal y como ha sido reconocidos internacionalmente. ${ }^{4}$

La discapacidad con la que se asocian es elevada, generan sufrimiento, problemas interpersonales y pueden durar muchos años, sobre todo cuando ocurren 
en la infancia y la adolescencia. Estos sucesos también generan pérdidas importantes para la sociedad, en términos de los días no laborados. Los resultados refuerzan, por tanto, la necesidad de ampliar la cobertura de tratamiento para atender las secuelas de la violencia, considerando las importantes variaciones de género y estadios de desarrollo. Asimismo, destacan, la relevancia de crear e implementar programas de prevención e intervención temprana, utilizando estrategias basadas en datos de investigación y monitoreadas constantemente.

\section{Referencias}

1. Saldívar G, Ramos L, Saltijeral MT. Inseguridad percibida, conductas de evitación y autoprotección en mujeres urbanas. Construcción y validación de escalas. Salud Ment 1996;19:27-34.

2. Caballero MA, Ramos L.Violencia: una revisión del tema dentro del marco de trabajo de investigación en el Instituto $\mathrm{N}$ acional de Psiquiatría. Salud Ment 2004; 27: 21-30.

3. US D epartment of Health and Human Services Mental Health.A report of the Surgeon General. United States, Rockville (MD): N ational Center for Mental Health Services, $N$ ational Institute of Health, $\mathrm{N}$ ational Institute of Mental Health; 1999.

4.W orld Health 0 rganization.W orld report on violence and health. Ginebra, Switzerland:W H O ;2002:346.

5. Instituto Ciudadano de Estudios sobre la Inseguridad,A.C. Proyección de la delincuencia del fuero común en México. Gaceta Informativa 10.

Disponible en: www.icesi.org.mx [2004, septiembre 15].

6. Instituto Ciudadano de Estudios sobre la Inseguridad, A.C. (ICESI). La percepción de inseguridad en las principales regiones urbanas de México. Gaceta Informativa 6. Disponible en: www.icesi.org.mx [2004, septiembre 15].

7. Instituto Ciudadano de Estudios sobre la Inseguridad,A.C. (ICESI). A nálisis de resultados relevantes de la EN SI-2. Gaceta Informativa 1.

Disponible en: www.icesi.org.mx [2004, septiembre 15].

8. $O$ laiz G, Rico B, D el Río A. Encuesta N acional sobre Violencia contra

las Mujeres 2003. México, DF: Secretaría de Salud, Instituto N acional de Salud Pública; 2004.

9. Russo N , Koss M, Ramos L. Rape: Cultural definitions and health outcomes. En: Usher JM, comp.W omen's health. Contemporary International Perspectives, Leicester England: BPS Books: 2000: 129-142. 10. Ramos L, Saldívar G, Medina-Mora ME, Rojas E. Prevalencia de abuso sexual en estudiantes y su relación con el consumo de drogas. Salud Publica Mex 1998; 40: 221-233.

11. Brodsky $B, O$ quendo $M, E l l i s ~ S, C$ retchen $L, H$ aas $G$, Malone $K$ et al. The relation of childhood abuse to impulsivity and suicidal behavior in adults with mayor depression. Am J Psychiatry 2001; 158:1871-1877. 12.Tapia R, Sepúlveda J, Medina-Mora ME, C araveo J, D e la Fuente JR. Prevalencia del síndrome de estrés postraumático en la población sobreviviente a un desastre natural. Salud Publica Mex 1987; 29:406-411. 13. American Psychiatric Association. D SM-IV: D iagnostic and statistical manual of mental disorders. $4^{\text {th }}$ edition. W ashington, DC:A merican Psychiatric Association; 1994.

14. Kilpatrick D G. Mental health needs. Trauma assessment and inter vention. En:The office for victims of crime (OVC). N ational Victim Assistance A cademy. Estados U nidos. D isponible en: www.ojp.usdoj.gov/ ovc/assist/nvaa2002 [2002, diciembre 4].
15. Resnick $H, A$ cierno $R$, Kilpatrick DG. Health impact of interpersonal violence. Section II: Medical and mental health outcomes. Behav Med 1997: 23: 65-79.

16. CíaAH.Trastorno por estrés postraumático. Diagnóstico y tratamiento integrado. Primera Edición. Buenos Aires: Imaginador 2001:472.

17. Kessler RC, Sonnega A, Bromet E, Hughes M, N elson C, Breslau N . Epidemiological risk factors for trauma and PTSD. En:Yehuda R, comp. Risk factors for posttraumatic stress disorder.W ashington, D.C.: American Psychiatric Press; 1995:23-59.

18. Medina-Mora ME, Borges G, Lara C, Benjet C, Blanco J, Fleiz C et al. Prevalencia de trastornos mentales y uso de servicios: resultados de la Encuesta $\mathrm{N}$ acional de Epidemiología Psiquiátrica en México. Salud Ment 2003; 26:1-16

19. W orld Health 0 rganization. International Consortium in Psychiatric Epidemiology. C ross-national comparisons of the prevalences and correlates of mental disorders. Bull W orld Health 0 rgan 2000;78:413426.

20. Kessler RC, Ustun TB.The W orld Mental Health (W MH) Survey Initiative Version of the W orld Health $\mathrm{O}$ rganization, Composite International Diagnostic Interview (CIDI). Int J Methods in Psychiatr Res 2004;13(2):93-121.

21. W orld Health $\mathrm{O}$ rganization. W orld Health $\mathrm{O}$ rganization. C omposite International Diagnostic Interview (CIDI) version 15. Ginebra, Suiza: W HO $; 2001$.

22. Sheehan DV.The anxiety disease. N ueva York (NY): Editorial Charles Scribner and Sons; 1983.

23. Frenk J, Lozano R, Gónzalez MA. Economía y salud: propuesta para el avance del sistema de salud en México. México DF: Fundación Mexicana para la Salud; 1999.

24. Research Triangle Institute. SUDDAN [computer program] Version 8.0.1. Research Triangle Park, N C: Research Triangle Institute; 2002. 25. Hosmer DW, Lemeshow S. Applied survival analysis. Regression modeling of time to event data. N ew York: Editorial John W iley and Sons; 1999.

26. Hosmer DW, Lemeshow S. A pplied logistic regression. 2da. edición. $\mathrm{N}$ ew York: Editorial John W iley and Sons; 2000.

27. Instituto $N$ acional de Estadística, G eografía e Informática. Encuesta $\mathrm{N}$ acional de Ingresos y $\mathrm{G}$ astos de los Hogares 2000. México: IN EGI; 2003.

28. Meichenbaum D.A Clinical handbook/practical therapist manual for assessing and treating adults with post-traumatic Stress Disorder (PTSD ). 0 ntario, Canada: Institute Press, W aterloo; 1994.

29. Terr LC. C hildhood traumas: An outline and overview. American Journal of Psychiatry 1991;148:10-20.

30.0 rganización Mundial de la Salud. Dando prioridad a las mujeres: recomendaciones éticas y de seguridad para la investigación sobre la violencia doméstica contra las mujeres. Programa mundial sobre pruebas científicas para las políticas de salud. Ginebra, Suiza: O MS; 1999. 31. Kaufman M.The construction of masculinity and the triad of men's violence. En:Toole LL, Schiffman JR, comp. Gender violence: Interdisciplinary perspectives. N ew York: University Press, 1997: 30-51. 32. Ramos L, González C. Bolaños F. Juventud, género y violencia. En: $\mathrm{N}$ ateras $\mathrm{DA}$, comp. Jóvenes, culturas e identidades urbanas. México, DF: UAM/Porrúa; 2002:415-432.

33. H eise L, Pitanguy J, Germain A.Violencia contra la mujer: la carga oculta sobre la salud. Programa mujer, salud y desarrollo. W ashington: O rganización Panamericana de la Salud; 1994.

34. American Psychological Association.Violence and the family: Report of the American Psychological Association Presidential Task Force on Violence and the Family.W ashington, DC:American Psychological Association; 1996.

35. Teicher MH. N eurobiología del maltrato en la infancia. Invest Ciencia 2002;308:59-67. 
36. American Psychiatric Association: D iagnostic and statistical manual of mental disorders, $3^{\text {rd }}$ edition.W ashington, DC:A merican Psychiatric Association; 1987.

37. Ramos L, Caballero MA.Violencia y delito. Efectos psicológicos y psicosociales. En: Calleja N , Gómez-Mitré G, comp. Psicología social: Investigación y aplicaciones en México. México: Fondo de Cultura Económica; 2001:380-397.

38. Yehuda R. Biological factors associated with susceptibility to posttraumatic stress disorder. C an J Psychiatry 1999; 44:317-326.

39. González RS. Comparación de psicoterapia de grupo vs psicoterapia individual en mujeres sobrevivientes de abuso sexual y/o incesto en la infancia. En:Torres M, comp.Violencia contra las mujeres en contextos urbanos y rurales. México: El Colegio de México, Programa Interdisciplinario de Estudios de la Mujer; 2004:271-303.
40. N atera G, Tiburcio M,Villatoro J. Marital violence and its relationship to excessive drinking in Mexico. Contemp D rug Problems Interdisciplin Q $1997 ; 24: 787-804$.

41. Medina-Mora ME, Berenzon S, N atera G. El papel del alcoholismo en las violencias. Gac Med Mex 1999;135:282-287.

42. González C, Ramos L,Vignau LE, RamírezVC. El abuso sexual y el intento suicida asociados con el malestar depresivo y la ideación suicida de los adolescentes. Salud Ment 2001; 24: 16-25.

43. Ramos L, Saltijeral T, Romero M, Caballero MA, Martínez N A.

Violencia sexual y problemas asociados en una muestra de usuarias de un centro de salud. Salud Publica Mex 2001; 43:182-191.

44.W HO W orld Mental Health Survey Consortium: Prevalence, severity, and unmet need for treatment of mental disorders in the W orld Health O rganization W orld Mental Health Surveys. JA MA 2004;2:2581-90. 


\section{Anexo \\ Sucesos Violentos}

Violación

Las dos preguntas siguientes son sobre violencia sexual. La primera se refiere a la violación. D efinimos como violación que alguien realice el acto sexual con usted o cualquier penetración con un dedo o con un objeto, cuando usted no desea que lo haga, ya sea mediante la fuerza o cuando era tan pequeño(a) que no podía saber lo que sucedía. ¿Alguna vez le ha ocurrido esto?

Abuso sexual

¿Alguna vez han abusado de usted o lo (la) han atacado sexualmente y lo han tocado de una forma que a usted le pareció inapropiada o cuando usted no lo deseaba?

Apaleado por padres

De niño ¿alguna vez recibió una paliza muy fuerte de sus padres o de las personas que lo criaron?

Apaleado por pareja

¿Alguna vez su esposo(a)/pareja le ha dado una paliza muy fuerte?

Apaleado por otros

¿Alguna vez otra persona le ha dado una paliza muy fuerte?

Guerra o conflictos

En la siguiente parte de la entrevista vamos a hablar de acontecimientos muy estresantes que pueden haberle ocurrido a lo largo de la vida. Para empezar, ¿ha participado usted en alguna guerra, como miembro del ejército o como miembro de una organización no militar? ¿H a sido un miembro de las fuerzas de paz, o colaborador humanitario en una zona en guerra 0 en un lugar donde hubiera una situación de terror entre la población, debido a conflictos políticos, raciales, religiosos o de cualquier otro tipo?

¿Alguna vez ha estado como civil desarmado en un lugar donde hubiera una guerra, una revolución, un golpe de estado militar o una invasión?

¿Alguna vez ha vivido como civil en un lugar donde hubiera una situación de terror entre la población civil por motivos políticos, raciales, religiosos o de cualquier otro tipo?

Secuestrado

¿Alguna vez ha sido secuestrado o ha estado prisionero?

Accidente tránsito/otro

¿Alguna vez ha estado expuesto a un tóxico o sustancia química peligrosa que pudiera provocarle daños graves?

¿Alguna vez se ha visto involucrado en un accidente de tránsito que hubiera podido causarle la muerte?

¿Alguna vez ha tenido algún accidente de otro tipo que hubiera podido causarle la muerte incluso en su lugar de trabajo?

Desastre natural/provocado

A Alguna vez se ha visto involucrado en un gran desastre natural como un huracán, un terremoto 0 inundaciones devastadoras?

¿Alguna vez se ha visto involucrado en un desastre causado por el hombre, como un incendio provocado por el hombre como un incendio provocado por un cigarrillo o en la explosión de una bomba?
Enfermedad

¿Ha tenido alguna enfermedad que hubiera podido causarle la muerte?

Atracado, amenazado con arma

¿Alguna vez lo han atracado, robado 0 amenazado con un arma?

Perseguido, amenazado con arma

¿Alguna vez ha sido perseguido o vigilado? Es decir, ¿lo han seguido 0 le han controlado sus actividades haciéndole sentir que corría un grave peligro?

Muerte de familiar cercano

¿Se le ha muerto algún familiar o amigo muy cercano de forma repentina 0 inesperada (por ejemplo, en un accidente, asesinato, suicidio 0 por un ataque al corazón a una edad temprana)?

Evento traumático a familiar

¿Algún familiar o amigo muy cercano ha pasado por una experiencia muy traumática, como por ejemplo un secuestro, violación o tortura?

Violencia intrafamiliar

Cuando era niño, ¿alguna vez vio peleas físicas en la casa como por ejemplo que su padre golpeara a su madre?

Vio que hirieran o mataran a alguien

¿A lguna vez ha visto que estuvieran hiriendo gravemente 0 matando a alguien, 0 ha visto inesperadamente un cadáver?

Hirió, mató accidentalmente a otro

Alguna vez ha hecho usted algo que provocara accidentalmente heridas graves o la muerte a una persona?

Hirió, mató intencionalmente a otro ¿Alguna vez ha herido gravemente, torturado o matado a alguien a propósito?

Testigo de atrocidades o matanzas ¿Alguna vez ha sido testigo de atrocidades o matanzas, como por ejemplo mutilaciones 0 asesinatos en masa?

Otro suceso traumático

¿Ha pasado por alguna otra situación muy traumática o que hubiera podido causarle la muerte que no le haya mencionado?

¿A lguna vez ha estado en situación de refugiado, es decir alguna vez ha tenido que abandonar su país de origen y refugiarse en el extranjero para escapar del peligro 0 de la persecución? 